TRANSACTIONS OF THE

AMERICAN MATHEMATICAL SOCIETY

Volume 363, Number 5, May 2011, Pages 2627-2640

S 0002-9947(2010)05151-7

Article electronically published on December 28, 2010

\title{
REPRESENTATIONS OF LOGMODULAR ALGEBRAS
}

\author{
VERN I. PAULSEN AND MRINAL RAGHUPATHI
}

\begin{abstract}
We study the question of whether or not contractive representations of logmodular algebras are completely contractive. We prove that a 2-contractive representation of a logmodular algebra extends to a positive map on the enveloping $C^{*}$-algebra, which we show generalizes a result of Foias and Suciu on uniform logmodular algebras. Our proof uses non-commutative operator space generalizations of classical results on 2-summing maps and semispectral measures. We establish some matrix factorization results for uniform logmodular algebras.
\end{abstract}

\section{INTRODUCTION}

Let $\mathcal{B}$ be a unital $C^{*}$-algebra and let $\mathcal{A} \subseteq \mathcal{B}$ be a unital subalgebra. By a representation of $\mathcal{A}$ on a Hilbert space $\mathcal{H}$ we mean a unital, contractive homomorphism, $\rho: \mathcal{A} \rightarrow B(\mathcal{H})$, where $B(\mathcal{H})$ denotes the $C^{*}$-algebra of bounded operators on $\mathcal{H}$. A representation of $\mathcal{A}$ is said to have a $\mathcal{B}$-dilation if there exists a Hilbert space $\mathcal{K}$, an isometry $V: \mathcal{H} \rightarrow \mathcal{K}$ and a unital $*$-homomorphism $\pi: \mathcal{B} \rightarrow B(\mathcal{K})$, such that $\rho(a)=V^{*} \pi(a) V$, for every $a \in \mathcal{A}$. By a famous result of Arveson 2, $\rho$ has a $\mathcal{B}$-dilation if and only if $\rho$ is a completely contractive map. For this reason there is a great deal of interest in results that imply that contractive representations are automatically completely contractive.

In particular, the Sz.-Nagy dilation theorem implies that contractive representations of the disk algebra are completely contractive, and it is proven in [14, 17] and $[8$ that contractive representations of many finite-dimensional CSL algebras, including algebras of block upper triangular matrices, are completely contractive.

However, it is still unknown if contractive representations of $H^{\infty}(\mathbb{D})$ or of the upper triangular operators on $\ell^{2}(\mathbb{N})$ are automatically completely contractive. It is known that weak*-continuous contractive representations of both these algebras are completely contractive. The case of $H^{\infty}(\mathbb{D})$ follows readily from Sz.-Nagy's theorem and the case of nest algebras is done in [18.

Both these algebras are examples of logmodular algebras, and it is still unknown if, more generally, contractive representations of logmodular algebras are always completely contractive.

Received by the editors June 2, 2008 and, in revised form, June 30, 2009.

2010 Mathematics Subject Classification. Primary 47L55; Secondary 47A67, 47A20.

Key words and phrases. Logmodular algebra, completely contractive, 2-summing, semispectral.

This research was supported in part by NSF grant DMS-0600191.

(C)2010 American Mathematical Society Reverts to public domain 28 years from publication 
If $\rho: \mathcal{A} \rightarrow B(\mathcal{H})$ is a representation, then we say that $\rho$ is $R_{n}$-contractive if $\rho_{1, n}: M_{1, n}(\mathcal{A}) \rightarrow B\left(\mathcal{H}^{(n)}, \mathcal{H}\right)$, defined by $\rho_{1, n}\left(\left(a_{1}, \ldots, a_{n}\right)\right)=\left(\rho\left(a_{1}\right), \ldots, \rho\left(a_{n}\right)\right)$, is contractive. The notion of $C_{n}$-contractive is defined by using columns instead of rows.

Foias and Suciu [10] proved, in modern terminology, that every $R_{2^{-}}$and $C_{2^{-}}$ contractive representation of a logmodular uniform algebra is automatically completely contractive. Thus, by their result, an $R_{2}$ - and $C_{2}$-contractive representation of $H^{\infty}(\mathbb{D})$ is completely contractive. However, it is still not known if every $R_{2^{-}}$and $C_{2}$-contractive representation of a general logmodular algebra is automatically completely contractive. In particular, it is not known if every $R_{2}$ - and $C_{2}$-contractive representation of the upper triangular operators on $\ell^{2}(\mathbb{N})$ is completely contractive.

In these notes we try to extend the result of Foias and Suciu to general logmodular algebras. We prove that if $\mathcal{A}$ is a logmodular subalgebra of a $C^{*}$-algebra $\mathcal{B}$, then every representation of $\mathcal{A}$ on $\mathcal{H}$ that is both $R_{2}$ - and $C_{2}$-contractive extends to a positive map from $\mathcal{B}$ to $B(\mathcal{H})$ and that this positive map is unique. The Foias-Suciu result then follows as a corollary.

A result of Pietsch, Pelczyński and Lindenstrauss [19, 13], on the existence of "dominating" measures for 2-summing maps, plays a central role in Foias-Suciu's proof. We give a new proof of this result using operator space methods and obtain a non-commutative analogue that we need for our generalization.

The second key element in the Foias-Suciu proof is Mlak's theory [15] of semispectral measures, and we obtain an analogue of this theory for non-commutative $C^{*}$-algebras.

Recent work of Blecher and Labuschagne [3, 4] has shown that many classical function-theoretic results on logmodular algebras extend to the "non-commutative" logmodular algebras. Their results consider only completely contractive representations and do not address the above problems, but we are able to use many of their ideas. For example, they prove that every completely contractive representation of an arbitrary logmodular algebra extends uniquely to a two-positive map on the $C^{*}$-algebra. In their case, the existence of a completely positive extension is automatic by Arveson's extension theorem, the issue is the uniqueness. In our case, the difficulty is the existence of the extension at all, while the techniques to prove uniqueness are essentially contained in their paper [3].

One limitation of the theory of logmodular algebras is the lack of many natural examples. We prove that the only logmodular CSL algebras of matrices are the block upper triangular matrices. Thus, by a result of [17] every contractive representation of these types of logmodular algebras is completely contractive. Possibly every logmodular subalgebra of the algebra of matrices is of this form. This result also indicates that possibly every logmodular completely distributive CSL algebra is a nest algebra.

\section{Definitions AND EXAMPles}

Let $X$ be a compact Hausdorff space. Let $C(X)$ denote the continuous complexvalued functions on $X$ and let $C(X)_{+}$denote the set of positive elements in $C(X)$. If $S \subset C(X)$, then let $\mathcal{S}^{-1}$ denote the set of elements in $\mathcal{S}$ that are invertible in $\mathcal{S}$. A logmodular algebra on $X$ is a subalgebra $\mathcal{A}$ of $C(X)$ such that the set $\left\{\log |a|: a \in \mathcal{A}^{-1}\right\}$ is dense in the real-valued functions on $C(X)$, which we denote by $C_{\mathbb{R}}(X)$. The following well-known fact is immediate. 
Lemma 2.1. $\mathcal{A} \subseteq C(X)$ is logmodular if and only if the set $\left\{|f|^{2}: f \in \mathcal{A}^{-1}\right\}$ is dense in the set of positive invertible functions on $X$.

This equivalent characterization of logmodularity motivates the following definition. Let $\mathcal{B}$ be a $C^{*}$-algebra and let $\mathcal{A}$ be a subalgebra. Following [3], we say that $\mathcal{A}$ is logmodular in $\mathcal{B}$ if the set $\left\{a^{*} a: a \in \mathcal{A}^{-1}\right\}$ is dense in $\mathcal{B}_{+}^{-1}$.

From the invertiblity of the $a \in \mathcal{A}^{-1}$, it follows that the set $\left\{a a^{*}: a \in \mathcal{A}^{-1}\right\}$ is also dense in $\mathcal{B}_{+}^{-1}$. Thus, if $\mathcal{A}$ is logmodular, then so is the subalgebra $\mathcal{A}^{*}$.

Some algebras possess a property called strong logmodularity or factorization. This means that every positive invertible element in $\mathcal{B}$ is of the form $a^{*} a$ for some $a \in \mathcal{A}^{-1}$.

Examples of logmodular algebras include the disk algebra $A(\mathbb{D}) \subseteq C(\mathbb{T})$, a consequence of the Fejér-Riesz theorem on the factorization of positive trigonometric polynomials. However, the disk algebra is not a logmodular subalgebra of $C\left(\mathbb{D}^{-}\right)$. More generally, $H^{\infty}(\mathbb{D})$ is (strongly) logmodular when viewed as a subalgebra of $L^{\infty}(\mathbb{T})$.

An algebra $\mathcal{A} \subseteq C(X)$ is called Dirichlet if and only if $\Re(\mathcal{A})$ is uniformly dense in $C_{\mathbb{R}}(X)$. This is equivalent to $\mathcal{A}+\overline{\mathcal{A}}$ being uniformly dense in $C(X)$. Every Dirichlet algebra $\mathcal{A}$ is logmodular on $C(X)$. However, the algebra $H^{\infty}$ is not Dirichlet. We refer the reader to [11, Section 3] for more about these examples.

If $\mathbb{A}:=\{z \in \mathbb{C}: 0<r \leq|z| \leq 1\}$ is a closed annulus, then the algebra of functions that are continuous on $\partial \mathbb{A}$ and analytic on the interior of $\mathbb{A}$ provides an example of an algebra that is not logmodular. However, a deep result of Agler shows that every representation of this algebra is completely contractive [1].

We now give some examples of non-commutative logmodular algebras. The Cholesky decomposition shows that the algebra of upper triangular matrices $\mathcal{T}_{n}$ is logmodular in $M_{n}$ and, more generally, nest subalgebras for countable nests are logmodular subalgebras of $B(\mathcal{H})$ since they have factorization by 2 .

We now prove that among a certain family of subalgebras of matrix algebras, the upper triangular matrices are essentially the only logmodular algebras. We let $M_{n}$ denote the $n \times n$ matrices and let $\mathcal{D}_{n} \subseteq M_{n}$ denote the subalgebra of diagonal matrices. Every subalgebra $\mathcal{A}$, such that $\mathcal{D}_{n} \subseteq \mathcal{A} \subseteq M_{n}$, is known to be the algebra of matrices left invariant by a commutative lattice of subspaces. Moreover, every subalgebra of $M_{n}$ that is left invariant by a commuting lattice of subspaces can be seen to be unitarily equivalent to such an algebra. Such algebras are called $C S L$ algebras. For further details on CSL algebras, see [7]. While the results below do not need this characterization, the characterization serves to put the result in perspective.

We call $\mathcal{A} \subseteq M_{n}$ an algebra of block upper triangular matrices if there are positive integers $n_{1}, \ldots, n_{k}$ such that $n=n_{1}+\ldots+n_{k}$, and $\mathcal{A}$ is the set of matrices of the form $\left(A_{i, j}\right)$, where $A_{i, j} \in M_{n_{i}, n_{j}}$ and $A_{i, j}=0$ whenever $i>j$.

Theorem 2.2. Let $\mathcal{D}_{n} \subseteq \mathcal{A} \subseteq M_{n}$. Then $\mathcal{A}$ is a logmodular subalgebra of $M_{n}$ if and only if after a permutation of the basis, $\mathcal{A}$ is an algebra of block upper triangular matrices.

Proof. If $\mathcal{A}$ is an algebra of block upper triangular matrices, then it contains the algebra of upper triangular matrices and hence is logmodular.

Conversely, assume that $\mathcal{A}$ is $\operatorname{logmodular}$. Since $\mathcal{D}_{n} \subseteq \mathcal{A}$, we easily see that $\mathcal{A}$ is the span of the matrix units $\left\{E_{i, j}\right\}$ that belong to $\mathcal{A}$. 
By a compactness argument, it follows that every positive $P \in M_{n}$ factors as $P=A^{*} A$, with $A \in \mathcal{A}$. Let $J$ denote the rank one positive matrix with all entries equal to 1 and write $J=A^{*} A$ with $A \in \mathcal{A}$. If we let $R_{1}, \ldots, R_{n}$ denote the rows of $A$, then $J=R_{1}^{*} R_{1}+\ldots+R_{n}^{*} R_{n}$ and each $R_{i}^{*} R_{i}$ is a rank one positive matrix. From this it follows that for each $i$, either $R_{i}^{*} R_{i}=0$ or $R_{i}^{*} R_{i}$ is a positive multiple of $J$.

Hence, we have a row $k$ such that $R_{k}^{*} R_{k}$ is a positive multiple of $J$ and so each entry of this row must be non-zero. Thus, $\left\{E_{k, j}: j=1, \ldots, n\right\} \subseteq \mathcal{A}$. Renumbering, we have that $\left\{E_{1, j}: j=1, \ldots, n\right\} \subseteq \mathcal{A}$. Now let $S=\left\{i: E_{i, 1} \in \mathcal{A}\right\}$. For $i \in S$ and any $j$, we have that $E_{i, j}=E_{i, 1} E_{1, j} \in \mathcal{A}$. If $i \notin S, j \in S$, then $E_{i, j} \notin \mathcal{A}$, or else, $E_{i, 1}=E_{i, j} E_{j, 1} \in \mathcal{A}$.

Thus, renumbering so that $S=\{1, \ldots, m\}$, we have that $E_{i, j} \in \mathcal{A}$, for all $1 \leq$ $i, j \leq m$, while $E_{i, j} \notin \mathcal{A}$, whenever, $m<i$ and $1 \leq j \leq m$, that is, $\mathcal{A}$ contains the entire upper left hand block. Relative to this decomposition, setting $p=n-m$, we have that $M_{n}=\left[\begin{array}{cc}M_{m} & M_{m, p} \\ M_{p, m} & M_{p}\end{array}\right]$ and

$$
\mathcal{A}=\left\{\left[\begin{array}{cc}
A & B \\
0 & C
\end{array}\right]: A \in M_{m}, B \in M_{m, p}, C \in \mathcal{A}_{1}\right\}
$$

where $\mathcal{A}_{1} \subseteq M_{p}$ is a subalgebra that contains the diagonal subalgebra $\mathcal{D}_{p}$.

Now if we let $P=\left[\begin{array}{cc}I_{m} & 0 \\ 0 & Q\end{array}\right]$, where $Q \in M_{p}$ is positive, then factoring, $P=A^{*} A$, with $A=\left[\begin{array}{cc}X & Y \\ 0 & Z\end{array}\right] \in \mathcal{A}$, we find that $I_{m}=X^{*} X, 0=X^{*} Y$ and $Q=Y^{*} Y+Z^{*} Z$. But the first equation implies that $X$ must be a unitary, and hence, $Y=0$, so that $Q=Z^{*} Z$, with $Z \in \mathcal{A}_{1}$.

Thus, $\mathcal{A}_{1}$ is a logmodular subalgebra of $M_{p}$ and we are done by induction.

Corollary 2.3. Let $\mathcal{D}_{n} \subseteq \mathcal{A} \subseteq M_{n}$. If $\mathcal{A}$ is a logmodular subalgebra of $M_{n}$, then every contractive representation of $\mathcal{A}$ is completely contractive.

Proof. In [17 it is proven that every contractive representation of an algebra of block upper triangular matrices is completely contractive.

We do not know if every logmodular subalgebra of $M_{n}$ is of this form, but we believe that this is the case. This result makes it natural to conjecture that every logmodular completely distributive CSL algebra is a nest algebra.

In [3, Proposition 4.3] it is shown that if $\mathcal{A}$ is logmodular on $\mathcal{B}$, then $C_{e}^{*}(\mathcal{A})=\mathcal{B}$, where $C_{e}^{*}(\mathcal{A})$ denotes Hamana's boundary algebra; thus $\mathcal{B}$ is already the smallest $C^{*}$-algebra that any completely isometric representation of $\mathcal{A}$ can generate.

\section{Non-COmmutative ANAlogues of some Classic ReSults}

In this section we prove analogues of a classic factorization result for 2-summing maps and obtain a non-commutative analogue of Mlak's [15] theory of semispectral measures.

We first turn our attention to 2-summing maps and their relation to completely bounded maps. Given a Hilbert space $\mathcal{H}$, we form two operator spaces, row Hilbert space, $\mathcal{H}_{r}=B(\mathcal{H}, \mathbb{C})$ and column Hilbert space, $\mathcal{H}_{c}=B(\mathbb{C}, \mathcal{H})$. For more details on the structure of these operator spaces, see [16]. 
Let $X$ be a compact Hausdorff space and let $\mathcal{V}$ be a subspace of $C(X)$. A map $\psi: \mathcal{V} \rightarrow \mathcal{H}$ is called 2-summing if there exists a constant $c$ such that

$$
\sum_{j=1}^{n}\left\|\psi\left(f_{j}\right)\right\|_{\mathcal{H}}^{2} \leq c^{2}\left\|\sum_{j=1}^{n}\left|f_{j}\right|^{2}\right\|_{\infty}
$$

for all $n \geq 1$ and $f_{1}, \ldots, f_{n} \in \mathcal{A}$. The least such constant $c$ is denoted by $a_{2}(\psi)$.

The following result follows from [20, Proposition 5.11] (see also [9, Theorem 5.7]) and the fact that, $a_{2}(\psi)=\pi_{2, c}(\psi)=\pi_{2, r}(\psi)$, but we provide a direct argument.

Lemma 3.1. Let $\mathcal{V} \subseteq C(X)$ be a subspace, let $\mathcal{H}$ be a Hilbert space and let $\psi$ : $\mathcal{V} \rightarrow \mathcal{H}$ be a linear map. Then the following are equivalent:

(1) $\psi: \mathcal{V} \rightarrow \mathcal{H}$ is 2-summing,

(2) $\psi: \mathcal{V} \rightarrow \mathcal{H}_{r}$ is completely bounded,

(3) $\psi: \mathcal{V} \rightarrow \mathcal{H}_{c}$ is completely bounded.

Moreover, in this case, the 2-summing norm $a_{2}(\psi)$ and the two completely bounded norms are equal.

Proof. We only prove the equivalence of (1) and (2) and the equality of the 2summing norm with the completely bounded norm into $\mathcal{H}_{r}$. The proof of the equivalence of (1) and (3) and the other equality is identical.

To this end, let $\left(h_{i, j}\right) \in M_{n}\left(\mathcal{H}_{r}\right)$ and note that

$$
\begin{aligned}
\left\|\left(h_{i, j}\right)\right\|^{2} & =\left\|\left(h_{i, j}\right)\left(h_{i, j}\right)^{*}\right\|=\left\|\left(\sum_{k=1}^{n}\left\langle h_{i, k}, h_{j, k}\right\rangle\right)\right\| \\
& =\sup \left\{\left|\sum_{i, j, k=1}^{n}\left\langle\lambda_{i} h_{i, k}, \mu_{j} h_{j, k}\right\rangle\right|: \sum_{i=1}^{n}\left|\lambda_{i}\right|^{2}=\sum_{j=1}^{n}\left|\mu_{j}\right|^{2}=1\right\} .
\end{aligned}
$$

Now,

(1) $\left\|\left(\psi\left(f_{i, j}\right)\right)\right\|^{2}$

$$
=\sup \left\{\left|\sum_{i, j, k=1}^{n}\left\langle\lambda_{i} \psi\left(f_{i, k}\right), \mu_{j} \psi\left(f_{j, k}\right)\right\rangle\right|: \sum_{i=1}^{n}\left|\lambda_{i}\right|^{2}=\sum_{j=1}^{n}\left|\mu_{j}\right|^{2}=1\right\} .
$$

Let $g_{k}=\sum_{i=1}^{n} \lambda_{i} f_{i, k}$ and $h_{k}=\sum_{j=1}^{n} \mu_{j} f_{j, k}$. We have that the quantity in (1) is smaller than

$$
\begin{aligned}
\sum_{k=1}^{n}\left|\left\langle\psi\left(g_{k}\right), \psi\left(h_{k}\right)\right\rangle\right| & \leq\left(\sum_{k=1}^{n}\left\|\psi\left(g_{k}\right)\right\|^{2}\right)^{1 / 2}\left(\sum_{k=1}^{n}\left\|\psi\left(h_{k}\right)\right\|^{2}\right)^{1 / 2} \\
& \leq a_{2}(\psi)^{2}\left\|\sum_{i=1}^{n}\left|g_{k}\right|^{2}\right\|^{1 / 2}\left\|\sum_{j=1}^{n}\left|h_{k}\right|^{2}\right\|^{1 / 2} .
\end{aligned}
$$


Now, $\left(g_{1}, \ldots, g_{n}\right)=\left(\lambda_{1}, \ldots, \lambda_{n}\right)\left(f_{i, j}\right)$ implies

$$
\begin{aligned}
\sum_{k=1}^{n}\left|g_{k}\right|^{2} & =\left(g_{1}, \ldots, g_{n}\right)\left(g_{1}, \ldots, g_{n}\right)^{*} \\
& =\left(\lambda_{1}, \ldots, \lambda_{n}\right)\left(f_{i, j}\right)\left(f_{i, j}\right)^{*}\left(\lambda_{1}, \ldots, \lambda_{n}\right)^{*} \\
& \leq\left(\lambda_{1}, \ldots, \lambda_{n}\right)\left\|\left(f_{i, j}\right)\right\|^{2}\left(\lambda_{1}, \ldots, \lambda_{n}\right)^{*} \leq\left\|\left(f_{i, j}\right)\right\|^{2}
\end{aligned}
$$

and similarly we can show $\sum_{k=1}^{n}\left|h_{k}\right|^{2} \leq\left\|\left(f_{i, j}\right)\right\|^{2}$.

Combining the above inequalities, we get

$$
\left\|\left(\psi\left(f_{i, j}\right)\right)\right\|^{2} \leq a_{2}(\psi)^{2}\left\|\left(f_{i, j}\right)\right\|^{2} .
$$

Hence, $\|\psi\|_{c b} \leq a_{2}(\psi)$, and so every 2-summing map is completely bounded into the row Hilbert space.

Conversely, if $\psi$ is completely bounded into the row Hilbert space, then we have

$$
\sum_{i=1}^{n}\left\|\psi\left(f_{i}\right)\right\|^{2}=\left\|\left(\psi\left(f_{1}\right), \ldots, \psi\left(f_{n}\right)\right)\right\|^{2} \leq\|\psi\|_{c b}^{2},
$$

and so $\|\psi\|_{c b} \geq a_{2}(\psi)$. Thus, the map $\psi$ is 2 -summing and the norms agree.

The result of [19, 13] on the existence of dominating measures is essential to the proof of Foias-Suciu [10. We now present a non-commutative version of this result from which the result of [19, 13] follows readily. Note that if $v \in \mathcal{H}_{r}=B(\mathcal{H}, \mathbb{C})$, then $v^{*} v \in B(\mathcal{H})$ is a rank one positive operator, while if $v \in \mathcal{H}_{c}$, then $v v^{*} \in B(\mathcal{H})$ is a rank one positive operator. The existence of the state in the following result is related to [20, Proposition 5.1].

Theorem 3.2. Let $\mathcal{B}$ be a unital $C^{*}$-algebra, let $\mathcal{X} \subseteq \mathcal{B}$ be an operator subspace and let $\mathcal{H}$ be a Hilbert space. If $\psi: \mathcal{X} \rightarrow \mathcal{H}_{r}=B(\mathcal{H}, \mathbb{C})$ (respectively, $\mathcal{H}_{c}=$ $B(\mathbb{C}, \mathcal{H})$ ) is completely contractive, then there exists a state $s: \mathcal{B} \rightarrow \mathbb{C}$ and a unital completely positive map $\Phi: \mathcal{B} \rightarrow B(\mathcal{H})$ such that for every $x \in \mathcal{X}$, we have that $\|\psi(x)\|^{2} \leq s\left(x x^{*}\right)$ and $\psi(x)^{*} \psi(x) \leq \Phi\left(x^{*} x\right)$ (respectively, $\|\psi(x)\|^{2} \leq s\left(x^{*} x\right)$ and $\left.\psi(x) \psi(x)^{*} \leq \Phi\left(x x^{*}\right)\right)$.

Proof. Writing an arbitrary operator $T \in B(\mathbb{C} \oplus \mathcal{H})$ as a $2 \times 2$ operator matrix, we have that $T=\left[\begin{array}{ll}\lambda & r \\ c & B\end{array}\right]$, where $\lambda \in \mathbb{C}, r \in \mathcal{H}_{r}, c \in \mathcal{H}_{c}$ and $B \in B(\mathcal{H})$. That is, we identify

$$
B(\mathbb{C} \oplus \mathcal{H})=\left[\begin{array}{cc}
\mathbb{C} & \mathcal{H}_{r} \\
\mathcal{H}_{c} & B(\mathcal{H})
\end{array}\right]
$$

Let

$$
\mathcal{S}_{\mathcal{X}}:=\left\{\left[\begin{array}{cc}
\lambda 1 & x \\
y^{*} & \mu 1
\end{array}\right]: \lambda, \mu \in \mathbb{C}, x, y \in \mathcal{X}\right\} \subseteq M_{2}(\mathcal{B})
$$

where 1 denotes the unit of $\mathcal{B}$. Since $\psi$ is completely contractive, the map $\Psi: \mathcal{S}_{\mathcal{X}} \rightarrow$ $B(\mathbb{C} \oplus \mathcal{H})$ given by

$$
\Psi\left(\left[\begin{array}{cc}
\lambda & x \\
y^{*} & \mu
\end{array}\right]\right)=\left[\begin{array}{cc}
\lambda & \psi(x) \\
\psi(y)^{*} & \mu I_{\mathcal{H}}
\end{array}\right]
$$

is completely positive by [16, Lemma 8.1]. Thus, by Arveson's extension theorem, we may extend $\Psi$ to a completely positive map on $M_{2}(\mathcal{B})$, which we still denote by $\Psi$. Thus, $\Psi: M_{2}(\mathcal{B}) \rightarrow B(\mathbb{C} \oplus \mathcal{H})$. By [16, Theorem 8.3], there exist two completely 
positive maps $\phi_{1}: \mathcal{B} \rightarrow \mathbb{C}$ and $\Phi_{2}: \mathcal{B} \rightarrow B(\mathcal{H})$ and a completely bounded map $u$ such that

$$
\Psi\left(\left[\begin{array}{ll}
b_{1,1} & b_{1,2} \\
b_{2,1} & b_{2,2}
\end{array}\right]\right)=\left[\begin{array}{cc}
\phi_{1}\left(b_{1,1}\right) & u\left(b_{1,2}\right) \\
u\left(b_{2,1}^{*}\right)^{*} & \Phi_{2}\left(b_{2,2}\right)
\end{array}\right] .
$$

For any $x \in \mathcal{X}$ the matrix $\left[\begin{array}{cc}x x^{*} & x \\ x^{*} & 1\end{array}\right] \geq 0$, which implies that $\left[\begin{array}{cc}\phi_{1}\left(x x^{*}\right) & \psi(x) \\ \psi(x)^{*} & I\end{array}\right]$ is positive, which yields

$$
\|\psi(x)\|^{2} \leq \phi_{1}\left(x x^{*}\right) .
$$

Since $\phi_{1}(1)=1$, we have that $s=\phi_{1}$ is the desired state.

On the other hand, $\left[\begin{array}{cc}1 & x \\ x^{*} & x^{*} x\end{array}\right] \geq 0$, which implies that $\left[\begin{array}{cc}1 & \psi(x) \\ \psi(x)^{*} & \Phi_{2}\left(x^{*} x\right)\end{array}\right]$ is positive, from which it follows that $\psi(x)^{*} \psi(x) \leq \Phi_{2}\left(x^{*} x\right)$, and $\Phi=\Phi_{2}$ is the desired unital completely positive map.

For the case where $\psi: X \rightarrow \mathcal{H}_{c}$ is completely contractive, we define

$$
\mathcal{S}:=\left\{\left[\begin{array}{ll}
\lambda & y^{*} \\
x & \mu I
\end{array}\right]: \lambda, \mu \in \mathbb{C}, x, y \in \mathcal{X}\right\}
$$

and $\Psi: \mathcal{S} \rightarrow B(\mathbb{C} \oplus \mathcal{H})$ via

$$
\Psi\left(\left[\begin{array}{cc}
\lambda & y^{*} \\
x & \mu I
\end{array}\right]\right)=\left[\begin{array}{cc}
\lambda & \psi(y)^{*} \\
\psi(x) & \mu I
\end{array}\right]
$$

and argue as above.

Corollary 3.3 (Lindenstrauss-Pelczyński, Pietsch). Let $\mathcal{V} \subseteq C(X)$ and let $\psi$ : $\mathcal{V} \rightarrow \mathcal{H}$ with $\mathcal{H}$ a Hilbert space. If $\psi$ is 2 -summing, then there exists a positive measure $\mu$ on $X$ with $\mu(X)=1$ such that $\|\psi(f)\|^{2} \leq a_{2}(\psi)^{2} \int_{X}|f|^{2} d \mu$.

We now turn our attention to obtaining $C^{*}$-algebraic generalizations of Mlak's theory of semispectral measures [15]. We shall need the following folklore result about quadratic forms on Hilbert spaces.

Proposition 3.4. Let $\mathcal{H}$ be a complex Hilbert space and let $\phi: \mathcal{H} \rightarrow \mathbb{C}$ be a bounded quadratic form, that is:

(1) $\phi(\lambda h)=|\lambda|^{2} \phi(h)$, for every $\lambda \in \mathbb{C}, h \in \mathcal{H}$,

(2) there exists a constant $C>0$, with $|\phi(h)| \leq C\|h\|^{2}$,

(3) $\phi(h+k)+\phi(h-k)=2 \phi(h)+2 \phi(k)$.

Then there exists a bounded operator, $T \in B(\mathcal{H})$, such that $\phi(h)=\langle T h, h\rangle$, for every $h \in \mathcal{H}$ and $\|T\| \leq 2 C$ and such a $T$ is unique.

Proof. This result is [16, Exercise 4.18], so we only sketch the proof. One defines a two-variable function $\psi: \mathcal{H} \times \mathcal{H} \rightarrow \mathbb{C}$ by polarization and then copies the steps of the simplified proof of the Jordan-von Neumann theorem to verify that $\psi$ is a bounded sesquilinear map.

Note that if we are given a unital $C^{*}$-algebra $\mathcal{B}$, a Hilbert space $\mathcal{H}$ and a positive map $\Phi: \mathcal{B} \rightarrow B(\mathcal{H})$, then for each vector $h \in \mathcal{H}$, we obtain a positive functional $\gamma(h)$ on $\mathcal{B}$ by setting $\gamma(h)(b)=\langle\Phi(b) h, h\rangle$. The following result characterizes such maps. We let $\mathcal{B}_{+}^{\dagger}$ denote the set of positive linear functionals on $\mathcal{B}$.

Theorem 3.5. Let $\mathcal{B}$ be a $C^{*}$-algebra, let $\mathcal{H}$ be a Hilbert space and let $\gamma: \mathcal{H} \rightarrow$ $\mathcal{B}_{+}^{\dagger}$ be a map. Then there exists a positive linear map $\Phi: \mathcal{B} \rightarrow B(\mathcal{H})$ such that 


$$
\begin{aligned}
& \gamma(h)(b)=\langle\Phi(b) h, h\rangle \text { for every } b \in \mathcal{B} \text { and every } h \in \mathcal{H} \text { if and only if } \\
& \text { (1) } \gamma(\lambda h)=|\lambda|^{2} \gamma(h) \text { for every } \lambda \in \mathbb{C} \text { and every } h \in \mathcal{H}, \\
& \text { (2) there is a constant } C \text { such that }\|\gamma(h)\| \leq C\|h\|^{2}, \text { for every } h \in \mathcal{H}, \\
& \text { (3) } \gamma(h+k)+\gamma(h-k)=2 \gamma(h)+2 \gamma(k), \text { for every } h, k \in \mathcal{H} \text {. }
\end{aligned}
$$

Moreover, if $\mathcal{B}$ is unital, then $\Phi$ is unital if and only if $\gamma(h)$ is a state for every $\|h\|=1$.

Proof. For each fixed $b \in \mathcal{B}$, we see that $h \rightarrow \gamma(h)(b)$ is a bounded quadratic form. Hence, there exists an operator $\Phi(b) \in B(\mathcal{H})$ such that $\gamma(h)(b)=\langle\Phi(b) h, h\rangle$. The linearity and positivity of the map $b \rightarrow \Phi(b)$ now follow from the fact that each $\gamma(h)$ is linear and positive. $h$.

Finally, $\Phi(I)=I$ if and only if $1=\langle\Phi(I) h, h\rangle=\gamma(h)(I)$, for every unit vector

Corollary 3.6 (Mlak's dilation theorem for semispectral measures). Let $X$ be a locally compact Hausdorff space, let $\mathcal{B}(X)$ be the $\sigma$-algebra of Borel measurable subsets of $X$ and let $\mathcal{H}$ be a Hilbert space and assume that for each $h \in \mathcal{H}$ we are given a positive, regular Borel measure $\mu_{h}$ on $X$, satisfying:

(1) $\mu_{\lambda h}=|\lambda|^{2} \mu_{h}$, for every $\lambda \in \mathbb{C}$ and every $h \in \mathcal{H}$,

(2) there is a constant $C$ such that $\mu_{h}(X) \leq C\|h\|^{2}$, for every $h \in \mathcal{H}$,

(3) $\mu_{h+k}+\mu_{h-k}=2 \mu_{h}+2 \mu_{k}$, for every $h, k \in \mathcal{H}$.

Then there exists a Hilbert space $\mathcal{K}$, a linear map $V: \mathcal{H} \rightarrow \mathcal{K}$ and a regular Borel projection-valued measure, $E: \mathcal{B}(X) \rightarrow B(\mathcal{K})$ such that for every Borel set, $B \subseteq X$, and every vector $h \in \mathcal{H}$, we have $\mu_{h}(B)=\langle E(B) V h, V h\rangle$.

Proof. By Theorem 3.5 there exists a positive map $\Phi: C_{0}(X) \rightarrow B(\mathcal{H})$, with $\langle\Phi(f) h, h\rangle=\int_{X} f d \mu_{h}$, for every $h \in \mathcal{H}$. By Stinespring's theorem [21, this positive map is completely positive. Therefore the map $\Phi$ dilates, that is, there exists a *-homomorphism $\pi: C_{0}(X) \rightarrow B(\mathcal{K})$ and a map $V: \mathcal{H} \rightarrow \mathcal{K}$ such that $\Phi(f)=$ $V^{*} \pi(f) V$. The result now follows by letting $E$ be the regular Borel projectionvalued measure such that $\pi(f)=\int_{X} f d E$.

It would be interesting to find a similar characterization of maps $\gamma$ of the form $\gamma(h)(b)=\langle\Phi(b) h, h\rangle$ such that $\Phi: \mathcal{B} \rightarrow B(\mathcal{H})$ is a completely positive map that did not reduce to a simple tautology.

\section{Representations of LOGMODUlar AlgEBRAS}

In this section we obtain our principal results on representations.

Given operator spaces $X$ and $Y$ and a linear map $\psi: X \rightarrow Y$, we call $\psi R_{n^{-}}$ contractive provided that

$$
\left\|\left(\psi\left(x_{1}\right), \ldots, \psi\left(x_{n}\right)\right)\right\| \leq\left\|\left(x_{1}, \ldots, x_{n}\right)\right\|,
$$

for every $x_{1}, \ldots, x_{n} \in X$ and row contractive if it is $R_{n}$-contractive for every $n$. We define $C_{n}$-contractive and column contractive, analogously.

Lemma 4.1. Let $X$ be an operator space and $\psi: X \rightarrow \mathcal{H}_{c}$ (respectively, $\mathcal{H}_{r}$ ) be a linear map. If $\psi$ is column contractive (respectively, row contractive), then $\psi$ is completely contractive. 
Proof. Begin by noting that we may identify $M_{m, n}\left(\mathcal{H}_{c}\right)$ with $B\left(\mathbb{C}^{n}, \mathcal{H}^{(m)}\right)$. Let $\left(x_{i, j}\right) \in M_{m, n}(X)$ and let

$$
\lambda=\left[\begin{array}{c}
\lambda_{1} \\
\vdots \\
\lambda_{n}
\end{array}\right] \in \mathbb{C}^{n}, \text { with } \sum_{j=1}^{n}\left|\lambda_{j}\right|^{2}=1
$$

Write $\left(x_{i, j}\right)=\left[C_{1}, \ldots, C_{n}\right], C_{j} \in M_{m, 1}(X)$. We have

$$
\left\|\left(\psi\left(x_{i, j}\right)\right) \lambda\right\|=\left\|\psi_{m, 1}\left(\sum_{j=1}^{n} \lambda_{j} C_{j}\right)\right\| \leq\left\|\sum_{j=1}^{n} \lambda_{j} C_{j}\right\|=\left\|\left(x_{i, j}\right) \lambda\right\| \leq\left\|\left(x_{i, j}\right)\right\| .
$$

Hence, $\psi$ is completely bounded and $\|\psi\|_{c b}=\|\psi\|_{c o l}$.

The row case is analogous.

Theorem 4.2. Let $\mathcal{B}$ be a $C^{*}$-algebra, let $\mathcal{A} \subseteq \mathcal{B}$ be logmodular and let $\rho: \mathcal{A} \rightarrow$ $B(\mathcal{H})$ be an $R_{2}$-contractive representation; then $\rho$ is row contractive. If $\rho$ is $C_{2}$ contractive, then $\rho$ is column contractive.

Proof. Let $a_{1}, \ldots, a_{n} \in \mathcal{A}$ with $\sum_{j=1}^{n} a_{j} a_{j}^{*} \leq 1$. We will prove our result by induction on $n$. The case $n=2$ is our hypothesis. Now let $\epsilon>0$ and choose $b \in \mathcal{A}^{-1}$ such that $\sum_{j=1}^{n-1} a_{j} a_{j}^{*}<b b^{*}<1+\epsilon-a_{n} a_{n}^{*}$, this choice being possible since $\mathcal{A}$ is logmodular. Set $b_{j}=b^{-1} a_{j}$ and note that $\sum_{j=1}^{n-1} b_{j} b_{j}^{*}=\sum_{j=1}^{n-1} b^{-1}\left(\sum_{j=1}^{n-1} a_{j} a_{j}^{*}\right) b^{*-1} \leq 1$. Hence the induction hypothesis yields $\sum_{j=1}^{n-1} \rho\left(b_{j}\right)^{*} \rho\left(b_{j}\right) \leq 1$ or, equivalently,

$$
\sum_{j=1}^{n-1} \rho\left(b^{-1} a_{j}\right) \rho\left(b^{-1} a_{j}\right)^{*}=\rho(b)^{-1}\left(\sum_{j=1}^{n-1} \rho\left(a_{j}\right) \rho\left(a_{j}\right)^{*}\right) \rho(b)^{*-1} \leq 1 .
$$

Applying the $R_{2}$ condition again we get

$$
\sum_{j=1}^{n} \rho\left(a_{j}\right) \rho\left(a_{j}\right)^{*} \leq \rho(b) \rho(b)^{*}+\rho\left(a_{n}\right) \rho\left(a_{n}\right)^{*} \leq 1+\epsilon .
$$

Since this is true for all $\epsilon$ we have our result.

The case of $C_{2}$-contractive is similar.

Proposition 4.3. Let $\mathcal{A} \subseteq \mathcal{B}$ be logmodular, let $\rho: \mathcal{A} \rightarrow B(\mathcal{H})$ be a unital homomorphism and let $h \in \mathcal{H}$ be a unit vector. If $\phi, \psi: \mathcal{B} \rightarrow \mathbb{C}$ are states such that $\|\rho(a) h\|^{2} \leq \phi\left(a^{*} a\right)$ and $\left\|\rho(a)^{*} h\right\|^{2} \leq \psi\left(a a^{*}\right)$ for all $a \in \mathcal{A}^{-1}$, then $\phi=\psi$.

Proof. For any $a \in \mathcal{A}^{-1}$, we have

$$
\begin{aligned}
1 & =\|h\|^{4}=\langle h, h\rangle^{2}=\left\langle\rho(a)^{-1} \rho(a) h, h\right\rangle^{2} \\
& =\left\langle\rho(a) h, \rho\left(a^{-1}\right)^{*} h\right\rangle^{2} \leq\|\rho(a) h\|^{2}\left\|\rho\left(a^{-1}\right)^{*} h\right\|^{2} \\
& \leq \phi\left(a^{*} a\right) \psi\left(a^{-1}\left(a^{*}\right)^{-1}\right)=\phi\left(a^{*} a\right) \psi\left(\left(a^{*} a\right)^{-1}\right) .
\end{aligned}
$$

For $x \in \mathcal{B}_{\text {sa }}$ and $t \in \mathbb{R}, e^{t x}$ is a positive invertible element of $\mathcal{B}$ and so the above inequality gives

$$
1 \leq \phi\left(e^{t x}\right) \psi\left(e^{-t x}\right)
$$

Let $u(t)=\phi\left(e^{t x}\right) \psi\left(e^{-t x}\right)$ and note that this is a differentiable function with a minimum at $t=0$. Taking the derivative and setting $t=0$ we get

$$
0=u^{\prime}(0)=\phi\left(x e^{t x}\right)-\left.\psi\left(x e^{-t x}\right)\right|_{t=0}=\phi(x)-\psi(x) .
$$


Since this holds for all selfadjoint elements in $\mathcal{B}$ and $\phi, \psi$ are selfadjoint maps we get $\phi=\psi$.

Note that the above result does not guarantee that such a state $\phi$ exists, nor does it guarantee that if $\phi$ exists, satisfying $\|\rho(a) h\|^{2} \leq \phi\left(a^{*} a\right)$, then $\left\|\rho(a)^{*}\right\| \leq \phi\left(a a^{*}\right)$.

Proposition 4.4. Let $\mathcal{A} \subseteq \mathcal{B}$ be logmodular and let $\rho: \mathcal{A} \rightarrow B(\mathcal{H})$ be a representation which is $R_{2}$-contractive and $C_{2}$-contractive. If $h \in \mathcal{H}$ is fixed, then there exists a positive linear functional $\phi: \mathcal{B} \rightarrow \mathbb{C}$ such that $\phi(1)=\|h\|^{2},\|\rho(a) h\|^{2} \leq \phi\left(a^{*} a\right)$, $\left\|\rho(a)^{*} h\right\|^{2} \leq \phi\left(a a^{*}\right)$ and $\phi(a)=\langle\rho(a) h, h\rangle$ and such $a \phi$ is unique.

Proof. It will be enough to consider the case where $\|h\|=1$, and prove that there exists a state $\phi$ as above, since uniqueness will follow by Proposition 4.3 . Since the map $a \mapsto \rho(a)$ is $C_{2}$-contractive, it follows by Theorem 4.2 that $a \mapsto \rho(a)$ is column contractive. Hence, by Lemma 4.1, the map $a \mapsto \rho(a) h$ is completely contractive as a map from $\mathcal{A}$ into the column Hilbert space $\mathcal{H}_{c}$. By Theorem 3.2, there exists a state $\phi_{1}$ such that $\|\rho(a) h\|^{2} \leq \phi_{1}\left(a^{*} a\right)$. Moreover, since $a \mapsto \rho(a)$ is $R_{2}$-contractive, the map $a^{*} \mapsto \rho(a)^{*} h$ is completely contractive as a map from $\mathcal{A}^{*}$ to the column Hilbert space $\mathcal{H}_{c}$. Applying Theorem 3.2 again yields a state such that $\left\|\rho(a)^{*} h\right\|^{2} \leq \phi_{2}\left(a a^{*}\right)$. From Proposition 4.3 we get that $\phi_{1}=\phi_{2}$.

Let $\lambda$ be a complex scalar and consider $\|\rho(a+\lambda) h\|^{2} \leq \phi\left((a+\lambda)^{*}(a+\lambda)\right)$. Expanding both sides and rearranging we get

$$
2 \Re(\lambda(\langle\rho(a) h, h\rangle-\phi(a))) \leq \phi\left(a^{*} a\right)-\|\rho(a) h\|^{2} .
$$

This quantity being bounded above for all $\lambda \in \mathbb{C}$ implies that $\langle\rho(a) h, h\rangle=\phi(a)$.

Lemma 4.5. Let $\mathcal{A} \subseteq \mathcal{B}$ be logmodular and let $\rho: \mathcal{A} \rightarrow B(\mathcal{H})$ be a representation that is $R_{2}$-contractive and $C_{2}$-contractive and for each $h \in \mathcal{H}$ let $\phi_{h}$ denote the unique positive linear functional as obtained in Proposition 4.4. Then for any vectors, $h, k \in \mathcal{H}, \phi_{h+k}+\phi_{h-k}=2 \phi_{h}+2 \phi_{k}$.

Proof. The proof is identical to the one outlined in [10, Lemma 3]. For any $a \in$ $(\mathcal{A})^{-1}$ we have

$$
\begin{aligned}
& 2\left(\|h\|^{2}+\|k\|^{2}\right) \\
= & \|h+k\|^{2}+\|h-k\|^{2} \\
= & \left\langle\rho(a)(h+k), \rho\left(a^{-1}\right)^{*}(h+k)\right\rangle+\left\langle\rho(a)(h-k), \rho\left(a^{-1}\right)^{*}(h-k)\right\rangle \\
\leq & \left(\|\rho(a)(h+k)\|^{2}+\|\rho(a)(h-k)\|^{2}\right)^{1 / 2} \\
& \times\left(\left\|\rho\left(a^{-1}\right)^{*}(h+k)\right\|^{2}+\left\|\rho\left(a^{-1}\right)^{*}(h-k)\right\|^{2}\right)^{1 / 2} \\
\leq & \left(\phi_{h+k}\left(a^{*} a\right)+\phi_{h-k}\left(a^{*} a\right)\right)^{1 / 2}\left(2 \phi_{h}\left(\left(a^{*} a\right)^{-1}\right)+2 \phi_{k}\left(\left(a^{*} a\right)^{-1}\right)\right)^{1 / 2} .
\end{aligned}
$$

Thus, for any $x=x^{*} \in \mathcal{B}$, and $t \in \mathbb{R}$, we have that

$$
4\left(\|h\|^{2}+\|k\|^{2}\right)^{2} \leq\left[\phi_{h+k}\left(e^{t x}\right)+\phi_{h-k}\left(e^{t x}\right)\right]\left[2 \phi_{h}\left(e^{-t x}\right)+2 \phi_{k}\left(e^{-t x}\right)\right] .
$$

Since $t=0$ is the minimum of the function on the right hand side, differentiating and evaluating at $t=0$ yields

$$
\begin{aligned}
0=\left(\phi_{h+k}(x)+\phi_{h-k}(x)\right)\left(2\|h\|^{2}\right. & \left.+2\|k\|^{2}\right) \\
& -\left(\|h+k\|^{2}+\|h-k\|^{2}\right)\left(2 \phi_{h}(x)+2 \phi_{k}(x)\right),
\end{aligned}
$$

from which the result follows. 
We can now state and prove our key result.

Theorem 4.6. Let $\mathcal{A} \subseteq \mathcal{B}$ be logmodular. If $\rho: \mathcal{A} \rightarrow B(\mathcal{H})$ is a unital homomorphism which is $R_{2}$-contractive and $C_{2}$-contractive, then there exists a positive map, $\Phi: \mathcal{B} \rightarrow B(\mathcal{H})$, extending $\rho$ and satisfying $\Phi\left(a^{*} a\right) \geq \rho(a)^{*} \rho(a), \Phi\left(a a^{*}\right) \geq \rho(a) \rho(a)^{*}$, for every $a \in \mathcal{A}$. Such a map is unique. Moreover, if $\Psi: \mathcal{B} \rightarrow B(\mathcal{H})$ is any completely positive map extending $\rho$, then $\Psi=\Phi$.

Proof. Let $\phi_{h}: \mathcal{B} \rightarrow \mathbb{C}, h \in \mathcal{H}$, be the family of positive functionals guaranteed by Proposition 4.4 If we fix $b \in \mathcal{B}$, then by Lemma 4.5 the map $h \mapsto \phi_{h}(b)$ is easily seen to be a bounded quadratic form on $\mathcal{H}$, and hence there exists a unique bounded operator $\Phi(b)$ with $\langle\Phi(b) h, h\rangle=\phi_{h}(b)$. The map $b \mapsto \Phi(b)$ is easily checked to be linear, positive and to satisfy the other conclusions stated in the theorem.

If $\Psi: \mathcal{B} \rightarrow B(\mathcal{H})$ is any positive map satisfying the above conclusions, then for each $h \in \mathcal{H}$, the positive linear maps, $\psi_{h}(b)=\langle\Psi(b) h, h\rangle$ satisfy the conclusions of Proposition 4.4, and hence $\psi_{h}(b)=\phi_{h}(b)$, for every $b$ and every $h$.

Finally, if $\Psi$ is a completely positive map extending $\rho$, then by the CauchySchwarz inequality for 2-positive maps, it will satisfy $\Psi\left(a^{*} a\right) \geq \rho(a)^{*} \rho(a)$ and $\Psi\left(a a^{*}\right) \geq \rho(a) \rho(a)^{*}$, and so we will have $\Psi=\Phi$, by the uniqueness result.

Thus, a completely positive extension of $\rho$ exists if and only if the positive extension that we have constructed is completely positive. We now recapture the main result of Foias-Suciu [10].

Corollary 4.7. Let $X$ be a compact, Hausdorff space, let $\mathcal{A} \subseteq C(X)$ be logmodular, let $\rho: \mathcal{A} \rightarrow B(\mathcal{H})$ be a unital homomorphism that is $R_{2}$-contractive and $C_{2}$-contractive. Then there exists a Hilbert space $\mathcal{K}$, an isometry $V: \mathcal{H} \rightarrow \mathcal{K}$, and a unital $*$-homomorphism $\pi: C(X) \rightarrow B(\mathcal{K})$ such that $\rho(a)=V^{*} \pi(a) V$, for every $a \in \mathcal{A}$. If the span of $\pi(C(X)) V \mathcal{H}$ is dense in $\mathcal{K}$, then this representation is unique up to unitary equivalence.

Proof. By Theorem 4.6, there exists a positive map $\Phi: C(X) \rightarrow B(\mathcal{H})$. By Stinespring's theorem 21] (see also [16]) such a map is automatically completely positive, and hence by Stinespring's dilation theorem [21] (see also [16]) the map $\Phi$ has a unique dilation of the above type.

Conversely, given any dilation of the above type, if we set $\Phi(b)=V^{*} \pi(b) V$, then $\Phi$ is a positive map satisfying the above conditions and hence is unique.

\section{MATRiX FACTORIZATION IN LOGMODUlaR ALGEBRAS}

In [16. Corollary 18.11], a necessary and sufficient condition is given for an operator algebra to have the property that every contractive representation is completely contractive in terms of a certain type of factorization. In particular, it is proven that a unital operator algebra $\mathcal{A}$ has the property that every contractive representation is completely contractive if and only if for every $n$ and for every $\left(a_{i, j}\right) \in M_{n}(\mathcal{A})$ with $\left\|\left(a_{i, j}\right)\right\|<1$, there exists some $m$, scalar matrices, $C_{0}, \ldots, C_{m}$ (of appropriate sizes) all with norm less than one and diagonal matrices, $D_{1}, \ldots, D_{m}$ (of appropriate sizes), whose diagonal entries are elements of the open unit ball of $\mathcal{A}$, such that

$$
\left(a_{i, j}\right)=C_{0} D_{1} C_{1} \cdots D_{m} C_{m}
$$


The term appropriate sizes means simply that the sizes are such that the product is defined. Combining this result with the result of Foias-Suciu yields some new results about uniform logmodular algebras.

Theorem 5.1. Let $\mathcal{A} \subseteq C(X)$ be a uniform logmodular subalgebra. Then every contractive representation of $\mathcal{A}$ is completely contractive if and only if for each $f_{1}, f_{2} \in \mathcal{A}$, with $\left|f_{1}\right|^{2}+\left|f_{2}\right|^{2}<1$, there exists an $m$ and scalar matrices, $C_{0}, \ldots, C_{m}$ (of appropriate sizes) all with norm less than one and diagonal matrices $D_{1}, \ldots, D_{m}$ (of appropriate sizes) whose diagonal entries are elements of the open unit ball of $\mathcal{A}$, such that

$$
\left[\begin{array}{l}
f_{1} \\
f_{2}
\end{array}\right]=C_{0} D_{1} C_{1} \cdots D_{m} C_{m}
$$

Proof. If $\rho$ is contractive and $D$ is a diagonal matrix in $M_{n}(\mathcal{A})$, then $\left\|\rho_{n}(D)\right\| \leq$ $\|D\|$. If the factorization condition is met, then we see, by applying $\rho$ to both sides of the above equation, that $\rho$ is $C_{2}$-contractive.

Note that whenever $\left[\begin{array}{l}f_{1} \\ f_{2}\end{array}\right]$ has a factorization as above, then

$$
\left[\begin{array}{ll}
f_{1} & f_{2}
\end{array}\right]=C_{m}^{t} D_{m} \cdots C_{1}^{t} D_{1} C_{0}^{t},
$$

where $C^{t}$ denotes the transpose. Since $\|C\|=\left\|C^{t}\right\|$ for scalar matrices, it follows from the same argument as in the previous paragraph that $\rho$ is also $R_{2}$-contractive. Hence, by the result of Foias-Suciu, the representation $\rho$ is completely contractive.

Conversely, if every contractive representation is completely contractive, then by the result cited above, every element of the unit ball of $M_{n}(\mathcal{A})$ has such a factorization. Thus, in particular, every element of the unit ball of $M_{2}(\mathcal{A})$ of the form $\left[\begin{array}{ll}f_{1} & 0 \\ f_{2} & 0\end{array}\right]$ can be factorized, from which the factorization in the statement of the theorem follows.

Of course, the columns of size two in the above theorem can be equally well replaced by rows of size two.

Theorem 5.2. Let $\mathcal{A} \subseteq C(X)$ be a uniform logmodular subalgebra. Then for every $n$ and every $\left(f_{i, j}\right)$ in the open unit ball of $M_{n}(\mathcal{A})$, there exists an $m$, scalar matrices, $C_{0}, \ldots, C_{m}$ (of appropriate sizes) all with norm less than one and block diagonal matrices, $D_{1}, \ldots, D_{m}$ (of appropriate sizes), whose direct summands are elements of the open unit ball of either $M_{2,1}(\mathcal{A})$ or $M_{1,2}(\mathcal{A})$, such that

$$
\left(f_{i, j}\right)=C_{0} D_{1} C_{1} \cdots D_{m} C_{m}
$$

Proof. Let $\mathcal{C}=\mathcal{A}$ as algebras but define a new family of matrix norms on $\mathcal{C}$ by the above factorization. That is, we declare the open unit ball of $M_{n}(\mathcal{C})$ to be the set of all matrices that can be expressed as products of scalar contractions and block diagonal matrices as above of norm less than one. Arguing as in [5] or in [16. Theorem 18.1], one shows that this definition yields a family of norms on $M_{n}(\mathcal{C})$ and that these norms satisfy the Blecher-Ruan-Sinclair [6] axioms to form an abstract operator algebra.

The proof will be complete if we can show that the identity map from $\mathcal{A}$ to $\mathcal{C}$ is a complete isometry.

Clearly, any matrix that is in the unit ball of $M_{n}(\mathcal{C})$ is also in the unit ball of $M_{n}(\mathcal{A})$. This shows that the identity map from $\mathcal{C}$ to $\mathcal{A}$ is completely contractive. 
Conversely, since we are allowing contractions from $M_{2,1}(\mathcal{A})$ in our definition of the norm, we have that

$$
\left\|\left[\begin{array}{l}
f_{1} \\
f_{2}
\end{array}\right]\right\|_{M_{2,1}(\mathcal{C})} \leq\left\|\left[\begin{array}{l}
f_{1} \\
f_{2}
\end{array}\right]\right\|_{M_{2,1}(\mathcal{A})} .
$$

Thus, the identity map is also an isometry from $M_{2,1}(\mathcal{A})$ to $M_{2,1}(\mathcal{C})$, that is, the identity map from $\mathcal{A}$ to $\mathcal{C}$ is $C_{2}$-contractive. Similarly, this identity map is $R_{2}$ contractive.

Since $\mathcal{C}$ is an abstract operator algebra, by the representation theorem of $[6$ (see [16, Corollary 16.7] for an alternate proof), it can be represented completely isometrically on a Hilbert space via some map $\rho: \mathcal{C} \rightarrow B(\mathcal{H})$. Regarding $\rho: \mathcal{A} \rightarrow$ $B(\mathcal{H})$, we see that $\rho$ is $C_{2}$-contractive and $R_{2}$-contractive on the uniform logmodular algebra $\mathcal{A}$, so by the Foias-Suciu result, it is a completely contractive map.

Thus, the identity map is completely contractive as a map from $\mathcal{A}$ to $\mathcal{C}$. As we saw earlier, the identity map is completely contractive from $\mathcal{C}$ to $\mathcal{A}$, and hence the identity map is a complete isometry. Thus, the original family of matrix norms on $\mathcal{A}$ is equal to the new family.

The above result can also be used as the basis of an approach to deciding whether or not the analogue of the Foias-Suciu result is true for logmodular subalgebras of non-commutative $C^{*}$-algebras. Given a logmodular subalgebra $\mathcal{A} \subseteq \mathcal{B}$, one can form a new operator algebra $\mathcal{C}$ by endowing $\mathcal{A}$ with a (possibly) new operator algebra norm as above, allowing the diagonals to be direct sums of block diagonal matrices where each block is either a $2 \times 1$ matrix or a $1 \times 2$ matrix over $\mathcal{A}$. The proof of the above result shows that every $\rho: \mathcal{A} \rightarrow B(\mathcal{H})$ that is both $R_{2^{-}}$and $C_{2}$-contractive will be completely contractive if and only if the identity map from $\mathcal{A}$ to $\mathcal{C}$ is a complete isometry. Thus, some progress could conceivably be made by studying the abstract operator algebra $\mathcal{C}$. These considerations motivate the following problem.

Question 5.3. Let $\mathcal{A} \subseteq \mathcal{B}$ be a logmodular subalgebra of a $C^{*}$-algebra and let $\mathcal{C}$ be the abstract operator algebra defined in the above paragraph. Is $\mathcal{C}$ a logmodular algebra?

\section{REFERENCES}

[1] Jim Agler, Rational dilation on an annulus, Ann. of Math. (2) 121 no. 3 (1985), 537-563. MR.794373 (87a:47007)

[2] W.B. Arveson, Interpolation problems in nest algebras, J. Funct. Anal. 20 (1975), 208-233. MR0383098 (52:3979)

[3] D. Blecher and L. Labuschagne, Logmodularity and isometries of operator algebras, Trans. Amer. Math. Soc. 355 (2003), 1621-1646. MR1946408 (2004c:46113)

[4] D. Blecher and L. Labuschagne, Noncommutative function theory and unique extensions, Studia Math. 178 (2) (2007), 177-195. MR2285438(2007m:46102)

[5] D.P. Blecher and V.I. Paulsen, Explicit constructions of universal operator algebras and applications to polynomial factorization, Proc. Amer. Math. Soc. 112 (1991), 262-292. MR1049839 (91j:46093)

[6] D.P. Blecher, Z.-J. Ruan and A.M. Sinclair, A characterization of operator algebras, J. Funct. Anal. 89 (1990), 188-201. MR 1040962 (91b:47098)

[7] K.R. Davidson, Nest Algebras, Pitman Research Notes in Mathematics 191 (Longman Scientific and Technical), London, 1988. MR 972978 (90f:47062)

[8] K.R. Davidson, V.I. Paulsen, and S.C. Power, Tree algebras, semidiscreteness, and dilation theory, Proc. London Math. Soc. 68 (3) (1994), 178-202. MR1243841 (94m:47087) 
[9] E. Effros and Z.-J. Ruan, Self-duality for the Haagerup tensor product and Hilbert space factorizations, J. Funct. Anal. 100 (1991), 257-284. MR1125226 (93f:46090)

[10] C. Foias and I. Suciu, On operator representation of logmodular algebras, Bulletin de l'Académie Polonaise des Sciences. Série des Sciences Mathématiques, Astronomiques et Physiques 16 (1968), 505-509 MR0238081 (38:6357)

[11] Kenneth Hoffman, Banach Spaces of Analytic Functions, Dover, New York, 1988. MR1102893 (92d:46066)

[12] Kenneth Hoffman, Analytic functions and Logmodular Banach algebras, Acta Math., 108 (1962), 271-317. MR0149330(26:6820)

[13] J. Lindenstrauss and A. Pelczyński, Absolutely summing operators in $L_{p}$ spaces and their applications, Studia Math. 29 (1968), 275-326. MR0231188 (37:6743)

[14] M.J. McAsey and P. Muhly, Representations of nonselfadjoint crossed products, Proc. London Math. Soc. 47 (3) (1983), 128-144. MR698930 (85a:46039)

[15] W. Mlak, Decompositions and extensions of operator-valued representations of function algebras, Acta Sci. Math.(Szeged) 30 (1969), 181-193. MR0285914(44:3131)

[16] V. Paulsen, Completely Bounded Maps and Operator Algebras, Cambridge Studies in Advanced Mathematics 78, Cambridge University Press, Cambridge, UK, 2002. MR 1976867 (2004c:46118)

[17] V. I. Paulsen and S.C. Power, Lifting theorems for nest algebras, J. Operator Theory 20 (1988), 311-317. MR.1004126 (90g:47010)

[18] V.I. Paulsen, S.C. Power and J.D. Ward, Semidiscreteness and dilation theory for nest algebras, J. Funct. Anal. 80 (1988), 76-87. MR.960224 (89h:47064)

[19] A. Pietsch, Absolut p-summierend Abbildungen in normierten Raumen, Studia Math. 28 (1967), 333-353. MR0216328 (35:7162)

[20] G. Pisier, The operator Hilbert space $O H$, complex interpolation and tensor norms, Memoirs Amer. Math. Soc., Vol 122, Number 585, July 1996. MR1342022 (97a:46024)

[21] W.F. Stinespring, Positive functions on $C^{*}$-algebras, Proc. Amer. Math. Soc. 6 (1955), 211216. MR0069403 (16:1033b)

Department of Mathematics, University of Houston, Houston, Texas 77204

E-mail address: vern@math.uh.edu

Department of Mathematics, Vanderbilt University, Nashville, Tennessee 37240

E-mail address: mrinal.raghupathi@vanderbilt.edu

URL: http://www.math.vanderbilt.edu/ mrinalr 